\title{
Câncer de colo uterino em idosas: revisão de literatura
}

\author{
Cervical cancer in elderly women: literature review
}

Cáncer de cuello uterino en mujeres de edad avanzada: revisión de literatura

Márcia Cristina Taveira Pucci Green ${ }^{1 *}$, Giovana Ferreira de Carvalho², Alessandra Bassinello Migliari², Anrela Urbiola Pereira². $^{2}$

\section{RESUMO}

Objetivo: Realizar uma revisão de literatura sobre a epidemiologia do câncer de colo uterino em idosas e a realização dos exames nessa população. Revisão bibliográfica: Em estudos no Brasil e em outros países do mundo, houve concordância que é um câncer mais incidente em mulheres pardas, casadas, com baixa escolaridade e com baixo nível socioeconômico. Os estágios mais avançados dessa doença estão relacionados à maior idade e o diagnóstico é dificultado devido características anatômicas específicas dessa população, aos padrões de comportamento, desinformação e falta de capacitação profissional para lidar com a população idosa. Considerações finais: Não há indicadores exatos sobre neoplasia cervical em idosas em razão das dificuldades na realização do diagnóstico e a má adesão das idosas a esse exame. Todavia, devido ao aumento da longevidade associada à maior exposição aos fatores de risco e maior propensão ao câncer, torna-se importante o direcionamento da anamnese e realização do exame ginecológico nessa faixa etária.

Palavras-chave: Neoplasias do colo do útero, Idosas, Programas de rastreamento, Sexualidade.

\section{ABSTRACT}

Objective: The aim of this paper is to carry out a literature review on the epidemiology of cervical cancer in elderly women and the performance of examination devoted to this gender group. Literature review: In all studies in Brazil and other countries there has been a consensus that cancer is more prevalent in brown, married women, with low level of education and from lower socioeconomic status. The more advanced stages of this disease are related to older age the diagnosis is hampered due to specific anatomical characteristics of this population, to the patterns of behavior, misinformation, and lack of professional training to deal with the elderly population. Final considerations: There are no exact indicators on cervical neoplasia in elderly women due to the difficulties in making the diagnosis and the poor adherence of the elderly women to this exam. However, taking into consideration the increase in longevity associated with greater exposure to risk factors and greater propensity to cancer, it is important to target anamnesis and realization of gynecological examination in this age group.

Key words: Cervical neoplasms, Elderly, Screening program, Sexuality.

\section{RESUMEN}

Objetivo: Realizar una revisión de la literatura sobre la epidemiología del cáncer de cuello uterino en mujeres de edad avanzada y la realización de exámenes en esta población. Revisión bibliográfica: En todos los estudios in Brasil y otros países del mundo, hubo consenso sobre el hecho de que el cáncer es más frecuente en mujeres negras, casadas, con bajo nivel de formación y estratos socioeconómicos más bajos. Las etapas más avanzadas de esta enfermedad están relacionadas con la edad avanzada y el diagnóstico se ve obstaculizado debido las características anatómicas específicas de esta población, a los patrones de comportamiento, desinformación y falta de capacitación profesional para tratar con la población de edad más avanzada. Consideraciones finales: No hay indicadores exactos sobre la neoplasia cervical en mujeres de edad más avanzada debido a las dificultades para hacer el diagnóstico y la escasa adherencia de las mujeres de edad avanzada este examen. Sin embargo, debido al aumento de la longevidad asociado con una mayor exposición a factores de riesgo y una mayor propensión al cáncer, es importante dirigir la anamnesis y realización el examen ginecológico a este grupo de edad.

Palabras clave: Neoplasias cervicales, Ancianos, Programas de detección, Sexualidad.

1Santa Casa de Misericórdia de Franca, Franca - SP. *E-mail: puccicolpo105@gmail.com

2Universidade de Franca (UNIFRAN), Franca - SP.

SUBMETIDO EM: 4/2020

ACEITO EM: 5/2020

PUBLICADO EM: 7/2020 


\section{INTRODUÇÃO}

O câncer de colo uterino (CCU) ou câncer cervical, ou ainda, neoplasia cervical, está entre as neoplasias malignas mais frequentes entre as mulheres brasileiras, principalmente na $4^{\mathrm{a}}$ e $5^{\mathrm{a}}$ décadas de vida. $O$ número de mortes em 2017, por essa causa, foi de 6.385 pacientes e a estimativa de novos casos em 2020 é de 16.590 (INCA, 2020). O principal fator de risco para o CCU é a infecção pelo Papiloma Vírus Humano (HPV), destacando os tipos 16 e 18, transmitido sexualmente, e que, juntamente com fatores do hospedeiro, altera as células do colo uterino causando lesões precursoras que podem mais tarde evoluir para neoplasia (HOFFMAN BL, et al., 2014; INCA, 2020).

O CCU é uma doença assintomática ou pouco sintomática em suas fases iniciais ou pré-invasivas, fazendo com que as mulheres não procurem atendimento médico nesse período. O rastreamento do CCU é muito importante tendo em vista que a doença pode existir sem apresentar sintomas no início. Esse é feito através do exame citológico, que, no Brasil, é recomendado realizar anualmente em mulheres dos 25 aos 64 anos de idade. No entanto, se dois exames anuais consecutivos forem normais, esse pode ser realizado, novamente, após três anos devido à evolução lenta da doença (FEBRASGO, 2017).

No câncer avançado, podem existir: sangramento vaginal intermitente ou após as relações sexuais, corrimento vaginal anormal (amarelo fétido ou sanguinolento) e dor abdominal com sintomas relacionados ao aparelho urinário ou intestinal. Tudo isso dependerá do grau de acometimento da doença (FEBRASGO, 2017).

Contudo, o aumento da longevidade, a persistência das práticas sexuais na senilidade com consequente aumento de novas infecções pelo HPV, associada à má adesão dessa faixa etária ao exame ginecológico, corroboram para o aparecimento do CCU também nas idosas. Assim, é importante não descartar o exame ginecológico acima dos 64 anos e individualizar a necessidade e uma possível coleta nessa população (LEITE BO, et al., 2019).

Logo, esta revisão de literatura tem como objetivo analisar dados epidemiológicos, destacar os índices de neoplasia cervical em idosas, no Brasil e em outros países, evidenciando empecilhos na sua realização.

\section{REVISÃO BIBLIOGRÁFICA}

\section{A população idosa e o câncer de colo uterino}

A população idosa está mais propícia a ter doenças crônico-degenerativas, assim como os diversos tipos de cânceres, incluindo os relacionados à sexualidade, como o CCU. Doenças crônicas como obesidade, hipertensão e diabetes reforçam a necessidade de rastreamento para CCU, além de incentivar cuidados preventivos (COFIE LE, et al., 2018). A grande parte dessas patologias são mais comuns em idosos que adultos jovens e aumentam a mortalidade (SARFATI D, et al., 2016).

Além do mais, tal população tem sua expectativa de vida aumentada e mantém atividades sexuais, ficando exposta ao risco de infecção pelo HPV, principal causa do CCU (LEITE BO, et al., 2019). Apesar dos homens permanecerem mais ativos que mulheres, $17 \%$ delas, entre 75 e 85 anos, referem também relacionar-se sexualmente, principalmente se possuem boas vivências relacionadas a isso. Há estudos relacionando o aumento da longevidade com a prática sexual e vários outros desvendando as causas das disfunções sexuais como fatores fisiológicos, psicológicos e sociais (FLEURY HJ e ABDO CHN, 2015).

Porém, apesar da modernidade e avanços da medicina e de outras áreas científicas, a prática sexual da população idosa ainda é um tabu e, desta forma, os idosos ainda são pouco informados sobre preservativos, doenças sexualmente transmissíveis e os tipos de cânceres associados (LEITE BO, et al., 2019).

\section{Estudos epidemiológicos}

Existem, no Brasil, diversos estudos observacionais de casos de CCU em variadas regiões do país, porém, estudos nacionais sobre a incidência por faixa etária, ainda são escassos. Sabe-se que se trata de um câncer raro antes dos 30 anos de idade, sua incidência maior é entre 45 e 50 anos e que, após a quarta década de vida, a mortalidade é progressiva (INCA, 2020). 
Em um estudo feito a partir da análise de registros hospitalares com 77.317 casos de CCU, o resultado constatado foi uma média de idade ao diagnóstico de 49,2 anos, sendo predominante em jovens, de cor parda, baixa escolaridade e casadas (THULER LDC, et al., 2012). Outro estudo descritivo, de 2018, avaliou 553 idosas com CCU no Maranhão e a média de idade encontrada já foi de 69,91, a maioria das participantes também com baixa escolaridade, pardas e casadas (MEDEIROS-VERZARO P e SARDINHA AHL, 2018).

Certos artigos trazem a porcentagem de idosas com CCU em determinado grupo de mulheres com a doença, um deles traz um resultado de 38,5\% entre 60 e 69 anos e de $61,5 \%$ acima de 70 anos, outro, 31\% de idosas com esse câncer (RIBEIRO JF, et al., 2016; MEIRA KC, et al., 2011). Um estudo realizado em Vitória, no Espírito Santo, encontrou um percentual de 70,9\% de casos de CCU entre as mulheres idosas analfabetas ou com ensino fundamental incompleto, destacando que essa doença está diretamente relacionada à idade avançada, à baixa escolaridade e à consequente negligência com exames de rastreamento, ao baixo nível socioeconômico e com a cor parda (CARVALHO MCMP e QUEIROX ABA, 2010).

Um dos determinantes do diagnóstico de CCU em estágios avançados é a idade. Para mulheres com idade entre 30 e 39 anos, a chance de ter doença avançada foi 10\% maior do que nas mulheres mais jovens, aumentando gradativamente até 2 vezes para a faixa etária de 60 anos ou mais. Tal conclusão foi registrada em um estudo, com 37.638 casos de CCU, a partir dos dados dos Registros Hospitalares de Câncer disponibilizados pelo Instituto Nacional de Câncer e pela Fundação Oncocentro de São Paulo, no período entre 01 de janeiro de 2000 e 31 de dezembro de 2009 (THULER LCS, 2014).

Há também um maior risco de aparecimento de carcinoma cervical invasivo nas mulheres acima de 60 anos em decorrência da dificuldade de reconhecimento precoce de anomalias displásicas devido a alterações celulares frequentes pós-menopausa. Todavia, mais de $70 \%$ das pacientes que possuem essas alterações e maiores de 40 anos têm DNA negativo para HPV (BRUNO MT, 2019).

Há ainda o hipoestrogenismo, que torna a junção escamo-colunar inacessível a visualização colposcópica e pode atrapalhar a coleta da citologia. Esse fator, somado às alterações benignas, atrofia, hipotrofia, reações inflamatórias levam à dificuldade própria do diagnóstico e surgimento de apreensão das pacientes idosas, mesmo que estas recorram à rotina ginecológica regularmente. Quanto a este problema propõe-se repetir o exame colpocitológico após tratamento tópico vaginal com estrógenos (BRUNO MT, 2019).

Todos esses dados sofrem de um viés, no Brasil, que é a má adesão ao rastreamento do CCU, principalmente pela população geriátrica. Vários fatores corroboram para isso como: a dificuldade de acesso aos serviços de saúde, os preconceitos da sociedade relacionados ao envelhecimento, a falta da disponibilidade de cuidadores que acompanhem as idosas ao serviço de saúde, pouco investimento em ações educativas, falta de capacitação dos profissionais de saúde para lidarem com a população idosa, além de medo, vergonha e desinformação por parte das pacientes (BATISTA AFC e CALDAS CP, 2017; SANTOS MS, et al., 2011).

\section{Câncer de colo uterino em outros países}

Um estudo realizado na região da África Subsaariana pretendia determinar o efeito da intervenção na educação em saúde. Incluiu mulheres de 11 a 70 anos, da região central de Gana, supondo que se as mulheres com 11 anos de idade fossem bem informadas sobre rastreamento e câncer de colo do útero, sobre os fatores de risco e estratégias de prevenção haveria uma redução de sua vulnerabilidade e das idosas. 0 estudo concluiu que após essas intervenções as pacientes começaram a avaliar a própria suscetibilidade à doença, diminuindo os riscos e aderindo aos programas de saúde (EBU NI, et al., 2019).

Em muitos países em desenvolvimento, o conhecimento das mulheres sobre câncer cervical e medidas preventivas é limitado. Além disso, a taxa de rastreamento do câncer do colo do útero é baixa em países de baixa renda. Por exemplo, estudos relataram que apenas 13-29\% das mulheres na Coréia do Norte e $28 \%$ no Gabão têm conhecimento do rastreamento do câncer do colo do útero e 15\% das mulheres na Índia, 26\% na Malásia, 32\% no Nepal e 36\% na Tailândia estão cientes da vacina contra o HPV (TOUCH S e OH J, 2018). 
Outro estudo realizado em Portugal mostra que houve declínio da mortalidade por câncer do colo do útero em todos os grupos etários, embora com estagnação deste indicador desde meados da década de 80 para grupos etários mais avançados. Estes resultados sugerem que deve haver investimento em transmitir informação relevante à população portuguesa, com o intuito de aumentar a sua participação em rastreios para CCU (TEIXEIRA AC, et al., 2019).

Em artigo sobre triagem de CCU na Alemanha expõe-se a recomendação da União Europeia e de diferentes órgãos nacionais, de se realizar o exame citológico de 20 a 25 anos e até 65 anos ou mais, reconhecendo a importância de uma faixa etária mais ampla de triagem (BRZOSKA P, et al., 2020). Já o Conselho de Estado Chinês propõe a realização desse exame dos 35 aos 59 anos, sendo que suas taxas de CCU têm aumentado, contrapondo os países em desenvolvimento. Isso porque no país a adesão ao rastreamento é muito baixa (ZHANG J, et al., 2019).

A Etiópia é um dos países com maior risco para esse CCU, principalmente em mulheres com 15 anos ou mais e a faixa etária alvo de detecção da doença nesse país é de 30 a 49 anos, porém pode ser realizado antes em determinados casos. Isso mostra diferentes condutas tendo em consideração o perfil populacional de cada país (GEBREGZIABHER D, et al., 2019).

$\mathrm{Na}$ França, o teste para rastreamento do CCU é recomendado a cada três anos para todas as mulheres sexualmente ativas com idades entre 25 e 65 anos (MIGNOT S, 2019), assim como Helsinque, na Finlândia. A capital do país realiza o rastreamento por tempo maior que o preconizado no restante do país (30-64 anos). Isto ocorre pela premissa que em muitos países de alta renda, o risco de câncer do colo do útero é elevado nas áreas urbanas. Um estudo anterior na Finlândia mostrou um efeito protetor prolongado do rastreamento nas idades de 55 a 69 e um forte indício de benefício adicional ao estender a idade superior a 65 para 0 rastreio (PANKAKOSKI M, 2019).

$O$ efeito do rastreamento nas idades de 55 a 69 pareceu igualmente eficaz como o realizado nas idades de 40 a 54 anos (LONNBERG S, 2012). Nos demais países europeus, a faixa etária para triagem do CCU varia de acordo com o país, entre 23 e 30 anos e 59 e 69 anos. Também há divergências quanto ao intervalo de triagem, pois a Islândia e a Noruega têm um intervalo de 3 anos, a Finlândia e a Estônia têm intervalos de 5 anos e a Suécia e a Dinamarca têm intervalos dependendo da idade (PARTANEN, 2019).

Na Austrália, o Programa Nacional de Triagem Cervical (NCSP) abrange atualmente mulheres de 25 a 74 anos que já foram sexualmente ativas, independente de terem sido vacinadas contra o HPV (ROESKE L, 2018). A citologia é recomendada a cada 2 anos em mulheres de 18 a 69 anos e, a partir de 2017, há uma triagem primária do HPV em 5 anos com genotipagem para HPV 16 e 18 em mulheres de 25 a 74 anos (CANFELL K, 2018).

O teste para deteç̧ão do HPV faz com que mais mulheres sejam incluídas na triagem e permite que as pacientes mais idosas, com os tipos oncogênicos potenciais recebam acompanhamento por pertencerem a um grupo de risco. As pacientes, na Austrália, são acompanhadas por registros de triagem cervical, realizados rotineiramente através de agendamentos, informados às pacientes três meses antes da data de validade do exame anterior (CANFELL K, 2018).

\section{O teste de HPV}

Um estudo de 2019 mostra o desempenho de dois testes disponível no mercado para a genotipagem do vírus HPV. O Array identifica 18 tipos de HPV de alto risco (entre eles o 16 e 18) e 11 tipos de HPV de baixo risco. Apresenta alta sensibilidade para o HPV 16 (maior que 90\%) e um pouco menor para o HPV 18 (em torno de 54\%). Já o Cobas 4800 HPV detecta o genótipo do HPV 16 e 18 separadamente, agrupando outros 12 tipos. Em resumo, identificou-se desempenho clínico alto semelhante nos dois testes, que são superiores à citologia oncótica e que podem, inclusive, proporcionar prognóstico (PESIC A, et al., 2019).

Há ainda outro artigo que traz a detecção do DNA do HPV na urina, referindo boa correlação entre o genótipo do vírus urinário e cervical, facilidade de amostragem e de acessibilidade pelas mulheres. Porém, existem desafios em relação à padronização das técnicas de extração do DNA e quanto ao armazenamento e processamento da urina (PATTYN J, et al., 2019). 
A Organização Pan Americana de Saúde e Organização Mundial da Saúde (OPAS/OMS) recomendam o teste de HPV como exame de triagem, pois tem uma sensibilidade maior que a citologia e detectam a presença do vírus oncogênico antes do desenvolvimento de lesões pré-cancerosas. Isso permitiria maior aceitação pelas mulheres em fazer o rastreamento do CCU e aumentaria a margem para seguimento e tratamento (OPAS, 2018).

Muitos programas de triagem aceitam o teste do HPV como complementar à citologia para melhor seleção e acompanhamento de mulheres com risco aumentado para CCU. Existem propostas para que o exame de detecção do DNA HPV faça parte do rastreamento primário, deixando a citologia para os casos com teste molecular positivo. No entanto, é preciso considerar o custo-benefício e os fatores psicossociais gerados em mulheres ao saberem que possuem o HPV, que este pode provocar lesões ou não ou mesmo provocar e regredir e que não há medicamentos específicos (SILVA ER, et al., 2015).

A realização do teste viral pode beneficiar mulheres com citologia alterada, acompanhando melhor as de maior risco para o câncer e, também, pode evitar procedimentos desnecessários nas que não possuem risco aumentado. O teste, de maneira isolada, é mais sensível e confiável, porém, não é mais específico que o exame citológico para detectar lesões precursoras do CCU. Assim, a colposcopia e citologia ainda são necessárias a fim de excluir falsos-positivos e falsos-negativos (SILVA ER, et al., 2015).

No Brasil, a FEBRASGO traz em seu protocolo a utilização do teste do HPV quando o exame citológico estiver alterado e para acompanhamento de lesões precursoras de CCU em graus mais avançados (2 e 3). É um co-teste que pode ser realizado em mulheres com 30 anos ou mais nessas situações. Se os dois exames forem negativos, pode-se repeti-los em 5 anos, se os dois forem positivos, é necessária colposcopia e, se o teste viral for negativo sem presença de lesões de alto grau em citologia, essa pode ser repetida em 1 ano (FEBRASGO, 2017). Esta sugestão ainda não está em utilização pelo Ministério da Saúde no Brasil.

Se o teste de HPV for negativo após o tratamento de lesões precursoras do CCU, o valor preditivo negativo deste é quase de cem por cento, ou seja, a chance de recidiva ou persistência da lesão é próxima de zero. Isso também contribui para direcionar melhor o rastreamento desse câncer evitando a realização excessiva de exames (FEBRASGO, 2017).

O Plano de Prevenção e Controle do Câncer Cervicouterino da OPAS (Organização Panamericana da Saúde) trabalha com três enfoques: vacinação, ampliação da cobertura de triagem e, tratamento oportuno das lesões pré-cancerosas e do câncer cervicouterino. A realização do teste para o HPV tem sido avaliada na busca por melhora da triagem, porém, de acordo com a OPAS e OMS, isso ainda exige compromisso político, um plano nacional para o combate ao câncer cervicouterino, recursos suficientes, diretrizes nacionais, capacitação dos profissionais de saúde e um bom sistema de monitoramento (OPAS, 2018).

Um estudo conseguiu apresentar técnicas de baixo custo para detectar o DNA do vírus HPV por meio de auto-coleta de amostras conseguindo rastrear mulheres de população rural na Bolívia (SURRIABRE P, et al., 2019). Na China, também foram obtidos bons resultados com a auto-amostragem de forma rápida e barata e as mulheres com o exame positivo para HPV de alto risco foram submetidas à colposcopia digital com um colposcópio portátil. As imagens com alta resolução permitiram o tratamento no mesmo dia (GOLDSTEIN A, et al., 2020).

Nos países de baixa e média renda (LMIC), as mulheres têm acesso limitado ao rastreamento do CCU. No Zimbábue, o sistema de auto-coleta para pacientes co-infectadas pelo HIV apresenta desempenho semelhante à coleta realizada por médicos, embora apresente uma sensibilidade inferior. A diferença é compensada pelo aumento da aceitabilidade e participação da triagem. (FITZPATRICK MB, et al., 2019). No México, também foi concluído que esse teste pode ser útil em ambientes de poucos recursos e com uma infraestrutura deficitária (PONCE EL, et al., 2011).

Em análise feita no Japão, com 17.284 participantes, adicionou-se o teste do HPV ao rastreamento e o diagnóstico de lesões pré-malignas de alto grau aumentou, assim como a adesão das mulheres ao programa (HASHIGUCHI M, et al., 2019). Maior sensibilidade para lesões pré-cancerosas pelo teste de HPV também foi constatada em estudo no Uruguai com 1.010 mulheres participantes, onde o exame detectou $100 \%$ das 
mesmas enquanto o citológico detectou 54\% de anormalidades não necessariamente malignas (RODRíGUEZ G, et al., 2019).

Diante dos resultados obtidos, pode-se inferir que a dificuldade em se identificar o câncer de colo de útero em mulheres idosas vem da junção de diversos fatores, que em resumo são as dificuldades de rastreamento, devido a questões culturais e sociais com dificuldade de adesão ao exame, e a própria mudança anatômica do colo, que com o envelhecimento passa a apresentar displasias não ocasionadas por HPV e mudança em sua arquitetura, por conta do hipoestrogenismo (LEITE BO, 2019; BRUNO MT, 2019).

\section{CONSIDERAÇÕES FINAIS}

Os dados relacionados ao câncer de colo uterino em idosas são variáveis de acordo com cada região e estudo. O aumento da expectativa de vida e da maior exposição aos fatores de risco, junto à má adesão ao rastreamento das neoplasias cervicais pela população idosa, são preocupações atuais. Logo, alternativas como individualizar as pacientes ou adicionar outros métodos de triagem como o teste para o HPV, até mesmo a auto-coleta, podem auxiliar, lembrando sempre que as ações educativas são fundamentais. Há necessidade de melhores estudos sobre como fazer um rastreamento adequado para o CCU, especialmente na população idosa.

\section{REFERÊNCIAS}

1. BATISTA AFC, CALDAS CP. Fatores que interferem na adesão da mulher idosa a programas de prevenção do câncer ginecológico. Revista de Enfermagem UERJ, Rio de Janeiro, 2017; 25:e21839.

2. BRUNO MT, et al. Management of ASC-US/HPV positive post-menopausal woman. Virology Journal, 2019; 16(39).

3. CANFELL K, et al. Protocol for Compass: a randomised controlled trial of primary HPV testing versus cytology screening for cervical cancer in HPV-unvaccinated and vaccinated women aged 25-69 years living in Australia. BMJ Open, 2018; 8:e016700.

4. CARVALHO MCMP, QUEIROX ABA. Lesões precursoras do câncer cervicouterino: evolução histórica e subsídios para consulta de enfermagem ginecológica. Esc Anna Nery (impr.), 2010; 14(3): 617-624.

5. COFIE LE, HIRTH JM, WONG R. Chronic comorbidities and cervical cancer screening and adherence among USborn and foreign-born women. Springer Link, 2018; 29(11): 1105 - 1113.

6. Controle do Câncer do Colo do Útero: conceito e magnitude. 2020. In: INSTITUTO NACIONAL DE CÂNCER (Brasil).

7. EBU NI, et al. Impact of health education intervention on knowledge and perception of cervical cancer and screening for women in Ghana. BMC Public Health, 2019; 19(1): 1505.

8. FITZPATRICK MB, et al. Community-based self-collected human papillomavirus screening in rural Zimbabwe. BMC Public Health, 2019; 19(1): 603.

9. FLEURYL HJ, ABDO CH. Sexualidade da mulher idosa. Diagnóstico e Tratamento, 2015; 20(3): 117-20.

10. GEBREGZIABHER D, et al. Correlates of cervical cancer screening uptake among female under graduate students of Aksum University, College of Health Sciences, Tigray, Ethiopia. BMC Res Notes, 2019; 12(1): 520.

11. GOLDSTEIN A, et al. Assessing the feasibility of a rapid, high-volume cervical cancer screening programme using HPV self-sampling and digital colposcopy in rural regions of Yunnan, China. BMJ Open, 2020; 10:e035153.

12. HASHIGUCHI M, et al. What Has Changed Since the Introduction of Human Papilomavirus Testing with the CytologyBased Cervical Cancer Screening System in Japan A Social Experimento. Acta Cytologica, 2019; 63: 385-390.

13. HOFFMAN BL, et al. Ginecologia de Williams. Porto Alegre: AMGH, 2014; 769p.

14. LEITE BO, et al. A Percepção das Mulheres Idosas Sobre o Exame de Prevenção do Câncer de Colo de Útero. Revista Online de Pesquisa, 2019; 11(5): 1347-1352.

15. LONNBERG S, et al. Auditoria de mortalidade do programa finlandês de rastreamento do câncer do colo do útero. International Journal of Cancer, 2012; 132(9): 3134-2140.

16. MEDEIROS-VERZARO P, SARDINHA AHL. Caracterização sociodemográfica e clínica de idosas com câncer do colo do útero. Revista de Salud Pública, 2018; 20(6): 718-724.

17. MEIRA KC, et al. Perfil de Mortalidade por Câncer do Colo do Útero no Município do Rio de Janeiro no Período 1999 2006. Revista Brasileira Cancerologia, 2011; 57(1): 7-14.

18. MIGNOT S, et al. Pap tests for cervical cancer screening test and contraception: analysis of data from the CONSTANCES cohort study. BMC Cancer, 2019; 19(1): 317.

19. OLHÊ L, et al. Papanicolaou na terceira idade: um desafio para a enfermagem. Revista Fafibe On-Line, 2013; VI (6): 78-86.

20. Organización Panamericana de la Salud. Experiencias con la implementación de programas de tamizaje de cáncer cervicouterino basados en las pruebas de VPH. Informe de reunión. Washington, DC.: OPS; 2018.

21. PANKAKOSKI M, et al. Effectiveness of cervical cancer screening at age $65-\mathrm{A}$ register-based cohort study. Plos One, 2019; 14(3):e0214486.

22. PARTANEN VM, et al. NordScreen - an interactive tool for presenting cervical cancer screening indicators in the Nordic countries. Acta Oncologica, 2019; 58(9): 1199-1204. 
23. PATTYN J, et al. Human papillomavirus detection in urine: Effect of a first-void urine collection device and timing of collection. Journal of Virological Methods, 2019; 264: 23-30.

24. PESIC A, et al. CIN2+ detection of the HPV DNA Array genotyping assay in comparison with the Cobas 4800 HPV test and cytology. Virology Journal, 2019; 16(92).

25. PONCE EL, et al. Self-collection of vaginal specimens for human papillomavirus testing in cervical cancer prevention (MARCH): a community-based randomised controlled trial. The Lancet, 2011; 378(9806): 1868-1873.

26. Rastreio, diagnóstico e tratamento do câncer de colo de útero. São Paulo: Federação Brasileira das Associações de Ginecologia e Obstetrícia (FEBRASGO), 2017.

27. RIBEIRO JF, et al. Aspectos sociodemograficos e clínico da mulher idosa com câncer de colo do útero. Revista de Epidemiologia e Controle de Infecção, Santa Cruz do Sul, 2016; 6(2): 63-67.

28. RODRíGUEZ G, et al. Tamizaje del cáncer de cuello uterino con test de HVP. Primeros resultados en el sistema público de Uruguay. Rev Méd Urug, 2019; 35(4): 267-280.

29. SANTOS MS, et al. Saberes e práticas de mulheres idosas na prevenção do câncer cérvico-uterino. Revista Brasileira de Enfermagem, Brasília, 2011; 64(3): 465-71.

30. SARFATI D, et al. The Impact of Comorbidity on Cancer and Its Treatment. CA: A Cancer Journal of Clinicians, 2016; 66(4): 337-350.

31. SILVA ER, et al. Diagnóstico molecular do papilomavírus humano por captura híbrida e reação em cadeia da polimerase. Femina, 2015; 43(4): 181-184.

32. SURRIABRE $P$, et al. Assessment of a new low-cost, PCR-based strategy for high-risk human papillomavirus DNA detection for cervical cancer prevention. BMC Infectious Diseases, 2019; 19(842).

33. TEIXEIRA AC, et al. Tendências do tempo na mortalidade por câncer do colo do útero em Portugal. Acta Médica Portuguesa, 2019; 32(6): 427-433.

34. TOUCH S, OH J. Knowledge, attitudes, and practices toward cervical cancer prevention among women in Kampong Speu Province, Cambodia. BMC Cancer, 2018; 18(294).

35. THULER LCS, et al. Determinantes do diagnóstico em estadio avançado do câncer do colo do útero no Brasil. Revista Brasileira de Ginecologia e Obstetrícia, 2014; 36(6): 237-243.

36. THULER LCS, et al. Perfil das Pacientes com Câncer do Colo do Útero no Brasil, 2000-2009: Estudo de Base Secundária. Revista Brasileira de Cancerologia, 2012; 58(3): 351-357.

37. ZHANG J, et al. Predicting Behavioral Intentions Related to Cervical Cancer Screening Using a Three-Level Model for the TPB and SCT in Nanjing, China. Int. J. Environ. Res. Public Health 2019; 16(19): 3575. 